\title{
A clinicomycological study on chronic dermatophytosis in a tertiary care hospital in North India: An observational study
}

\author{
Rabiya Bashir'1, Naina Kala Dogra', Bella Mahajan²
}

${ }^{1}$ Post-Graduate Departments of Dermatology, Government Medical College, Jammu, India, ${ }^{2}$ Microbiology, Government Medical College, Jammu, India

Corresponding author: Rabiya Bashir, MD, E-mail: rabiyabashir72@gmail.com

\begin{abstract}
Background: Chronic dermatophytosis is a considerable challenge in routine clinical practice. There is, however, scarce information available in the literature on its extent and characteristics. Aim: The aim of this study was to evaluate the host-related factors of chronic dermatophytosis and to identify the common fungal isolates. Methods: The study enrolled a total of 145 cases of chronic dermatophytosis attending the out-patient department of a tertiary care hospital in Jammu from November 2017 through October 2018. A detailed history was taken, followed by a clinical examination and investigations such as routine baseline investigations, an absolute eosinophil count, a wet mount for direct microscopy, and a fungal culture. Results: The most common presentation was tinea corporis with tinea cruris (33.1\%), followed by tinea corporis alone. The majority of the patients (54.5\%) had more than $20 \%$ of the body surface area involved. Most of the patients were manual workers $(\mathrm{n}=44 ; 30.3 \%)$. The number of hours of sun exposure varied between 1 to 8.5 hours (mean \pm SD: $3.53 \pm 1.75 \mathrm{~h}$ ). The fungal culture was positive in $65(44.8 \%)$ patients. The most frequent isolates were Trichophyton mentagrophytes (53.8\%), followed by Trichophyton rubrum (38.5\%). Conclusion: We found Trichophyton mentagrophytes the predominant pathogen in chronic dermatophytosis, followed by Trichophyton rubrum, which demonstrates a changing trend as far as the causative organism is considered. Besides, various risk factors for chronicity such as prolonged sun exposure, lack of proper hygiene, wearing tight-fitting synthetic clothes, the use of topical steroids, and non-compliance to treatment were identified.
\end{abstract}

Key words: Chronic dermatophytosis; Trichophyton mentagrophytes; Topical steroids

\section{INTRODUCTION}

The dermatophytes are a group of closely related fungi with the capacity to invade keratinized tissue to produce infection: dermatophytosis. The etiologic agents of dermatophytosis include three anamorphic (asexual) genera: Epidermophyton, Microsporum, and Trichophyton [1].

We are witnessing, nowadays, a tremendous increase in the number of cases of chronic and recurrent dermatophytosis [2]. This pattern of dermatophytosis is also reported in other parts of the world, especially the tropics [3]. Although there is no standard definition of chronic dermatophytosis, the term refers to patients who have been suffering from the disease for more than six months to one year, with or without recurrence, in spite of being treated [2]. In view of the lack of recent studies on the various host and pathogen related factors contributing to chronic dermatophytosis, this observational study was conducted.

\section{METHODS}

\section{Study Design and Setting}

This was an observational study conducted at the Postgraduate Department of Dermatology of a tertiary

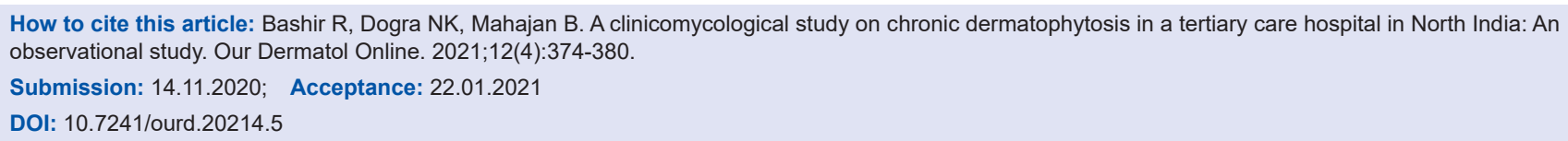


care hospital in North India from November 2017 through October 2018. After obtaining approval from the institutional ethical committee, patients reporting to the Dermatology OPD were deemed eligible. The diagnosis of dermatophytosis was clinical. A written informed consent was taken from all the patients.

\section{Inclusion Criteria}

Included were clinically diagnosed cases of dermatophytosis with the duration of the disease of at least six months, with or without recurrence.

\section{Exclusion Criteria}

Excluded were:

1. Patients suffering from dermatophytosis for less than six months.

2. Patients suffering from onychomycosis without lesions of tinea on other body sites.

3. Pregnant and lactating women.

\section{Patient Characteristics}

A detailed history was taken on the following variables and was recorded in a predesigned proforma: age, gender, duration of disease, treatment history, family history, personal history, personal hygiene, type of clothing, fomite sharing, overcrowding, socioeconomic status, occupational history particularly regarding working in hot and humid conditions, other systemic or cutaneous diseases such as immunodeficiency states or atopy, and any concomitant drug intake known to cause interactions with antifungals.

A detailed general, physical, systemic, and cutaneous examination was performed. The assessment of lesions of dermatophytosis included the site of involvement and the percentage of body surface involvement. Accordingly, the disease was classified as tinea capitis, tinea corporis, tinea cruris, tinea barbae, tinea faciei, tinea manuum, tinea pedis, and tinea unguium.

Investigations such as complete blood count (CBC), a kidney function test (KFT), a liver function test (LFT), absolute eosinophil count, and blood sugar fasting were performed. HIV serology was performed only for highrisk patients after proper counseling.

\section{Sample Collection for Mycological Study}

After properly cleaning the lesion with a spirit swab, samples were collected by scraping the active edges with a sterile scalpel blade. Hairs were taken in case of tinea capitis. The samples obtained were taken to the laboratory in clean black paper envelopes. $\mathrm{KOH}$ wet mount preparations were made and examined under the microscope against the presence of fungal hyphae. The specimens were inoculated into test tubes with Sabouraud dextrose agar with cycloheximide $(0.05 \mathrm{~g} / \mathrm{L})$ and chloramphenicol $(0.005 \mathrm{~g} / \mathrm{L})$ and incubated at $28^{\circ} \mathrm{C}$ for at least four weeks before labeling as negative. Species identification was performed by gross morphology of the fungal colonies, pigmentation, and microscopy with a Lactophenol cotton blue mount.

\section{Statistical Analysis}

Initially, the data was entered in a predesigned proforma and, subsequently, a database was created with Microsoft Excel for the purpose of data analysis. The data was reported as mean values and percentages as was appropriate for the quantitative and qualitative variables, respectively.

\section{RESULTS}

The mean age of the study subjects was $34.7 \pm 12.9$ years, ranging between 9 to 70 years. The most cases were in the age group $30-49$ years $(n=70 ; 48.3 \%)$. The male-to-female ratio was 1.26:1. The mean duration of the disease was $13.4 \pm 6.18$ months, ranging from 7 to 36 months. With most patients, the duration of the disease ranged from 10 to 19 months $(n=80 ; 55.2 \%)$.

Most of the patients were manual workers ( $n=44$; $30.3 \%$ ). The number of hours of sun exposure varied between 1 to 8.5 hours $(3.53 \pm 1.75$ hours). Around 99 $(68.3 \%)$ patients were exposed to more than 2.5 hours of sunlight a day.

Personal hygiene was average in most of the patients $(\mathrm{n}=81 ; 55.9 \%)$. The most preferred type of cloth fabric was synthetic, which was used by more than half of the patients $(50.4 \%)$. A history of fomite sharing was present in the majority of the patients $(74.5 \%)$.

A majority of the patients (55.2\%) lived in overcrowded conditions. Almost all socioeconomic classes were affected, with the upper middle class ( $\mathrm{n}=40 ; 27.6 \%$ ), upper lower class ( $\mathrm{n}=38 ; 26.2 \%$ ), and lower middle class $(\mathrm{n}=37 ; 25.5 \%)$ almost equally affected, followed by the lower class $(\mathrm{n}=24 ; 16.5 \%)$ and upper class $(\mathrm{n}=6 ; 4.1 \%)$. 
Comorbidities were present in 34 patients. Diabetes was present in $12(8.3 \%)$ patients, followed by hypertension in $11(5.5 \%)$ patients, atopic dermatitis and bronchial asthma in $3(2.1 \%)$ patients each, and anemia in $2(1.4 \%)$ patients. Osteoarthritis, palmoplantar psoriasis, and pemphigus vulgaris were present in $1(0.7 \%)$ patient each.

A family history of dermatophytosis was present in $63(43.5 \%)$ patients. Only some patients had a family history of atopy $(\mathrm{n}=16 ; 11.0 \%)$.

All patients had used topical antifungals. A majority $(\mathrm{n}=114 ; 78.6 \%)$ had used steroid-containing antifungal creams, and most $(\mathrm{n}=106 ; 73.1 \%)$ had used systemic antifungals, such as itraconazole, terbinafine, fluconazole, and griseofulvin. However, adequate antifungal use was noted only in $56(38.6 \%)$ patients. Additionally, the use of systemic corticosteroids was noted in $9(6.21 \%)$ patients.

More than half of the patients (54.5\%) had more than $20 \%$ of the body surface area involvement, and a majority $(\mathrm{n}=105 ; 72.4 \%)$ had multiple-site involvement, followed by tinea corporis alone $(n=23 ; 15.9 \%)$ and tinea cruris alone $(\mathrm{n}=17 ; 11.7 \%)$ (Fig. 1). The most common presentation was tinea corporis with tinea cruris (33.1\%), followed by tinea corporis alone. Among those with multiple-site involvement, a significant number had tinea faciei (14.48\%) and tinea unguium (13.10\%) (Figs. 2 - 3). Various atypical presentations such as eczematous, pustular, pseudoimbricata, etc., were also seen in several patients (Fig. 4).

The absolute eosinophil count was raised (> 450 cells $/ \mu \mathrm{L})$ in $32(26.1 \%)$ patients. Out of the 145 patients, $20(13.79 \%)$ were tested for an HIV infection but were all negative.

$\mathrm{KOH}$ scraping was positive for fungal hyphae in $91.3 \%$ of the patients.

A fungal culture was positive in 65 (44.8\%) patients. The most frequent isolate was Trichophyton mentagrophytes $(\mathrm{n}=35 ; 53.9 \%)$ (Figs. 5-6), followed by Trichophyton rubrum ( $\mathrm{n}=25 ; 38.5 \%$ ), Trichophyton tonsurans $(\mathrm{n}=3 ; 4.6 \%)$, Microsporum canis $(\mathrm{n}=1 ; 1.5 \%)$, and Trichophyton verrucosum $(\mathrm{n}=1 ; 1.5 \%)$.

\section{DISCUSSION}

We studied a total of 145 patients with chronic dermatophytosis. The most commonly affected

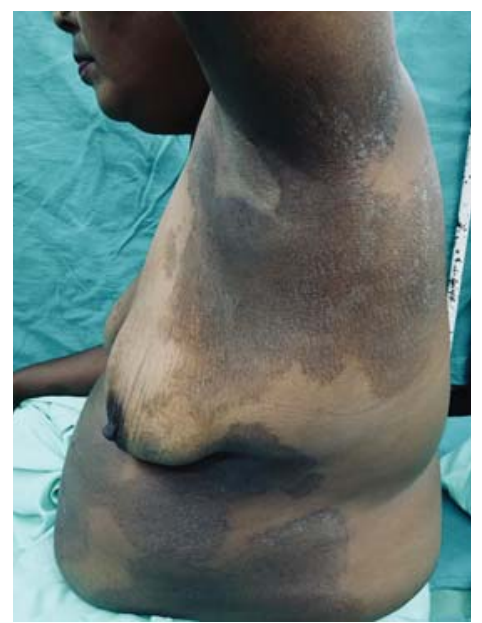

Figure 1: Extensive tinea corporis and tinea axillaris caused by $T$. mentagrophytes in a patient with bronchial asthma on oral steroids.

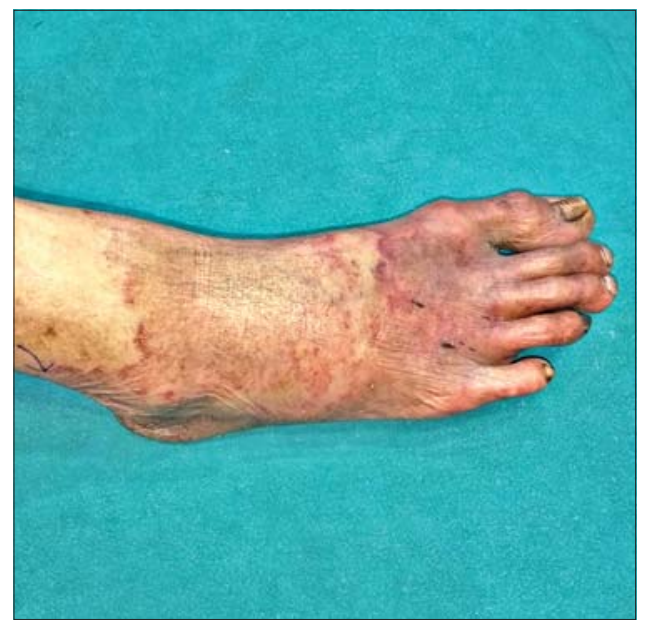

Figure 2: Tinea pedis extending to the dorsum with onychomycosis in a diabetic patient.

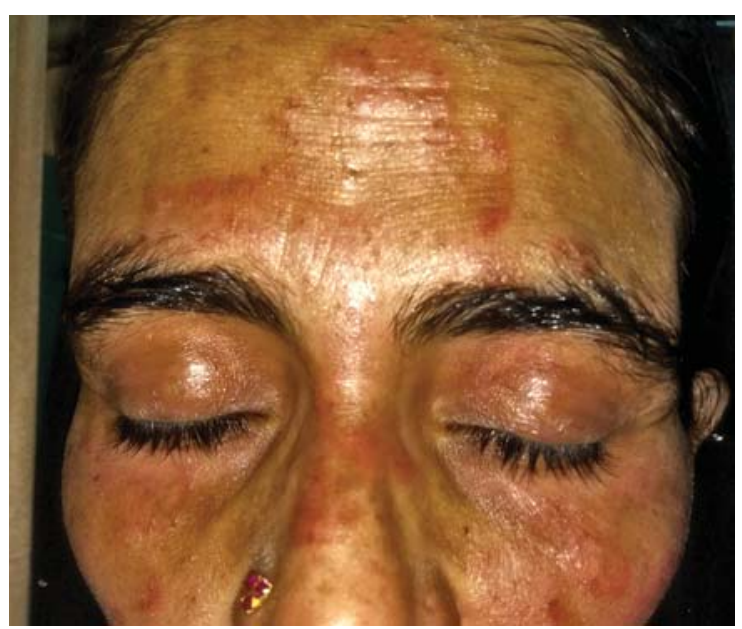

Figure 3: Tinea faciei in an adult.

age group was 30-49 years old. These results are in accordance with a study by Sivaprakasam and Govindan [4]. Also, in a study by Vineetha et al. [5], 


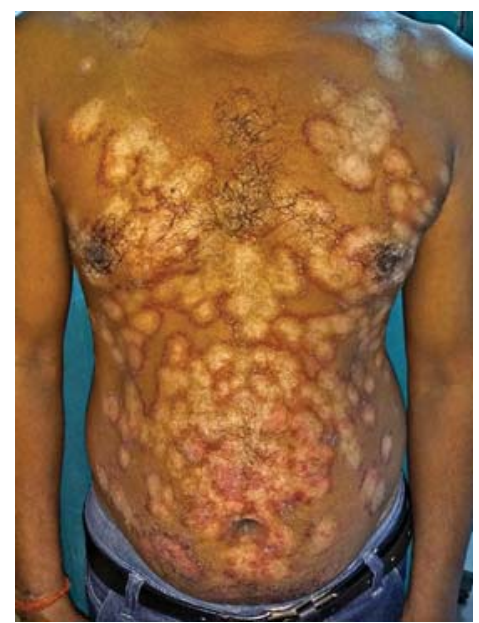

Figure 4: Atypical presentation of tinea corporis in a patient with steroid abuse: multiple coalescent annular lesions with central hypopigmentation.

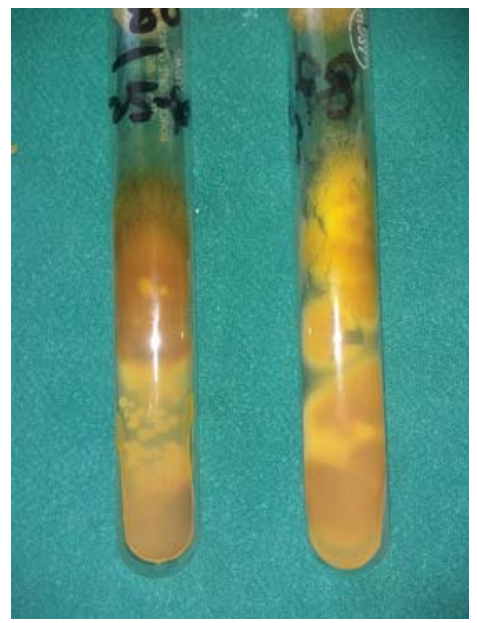

Figure 5: Obverse and reverse of macroscopic colony morphology of T. mentagrophytes.

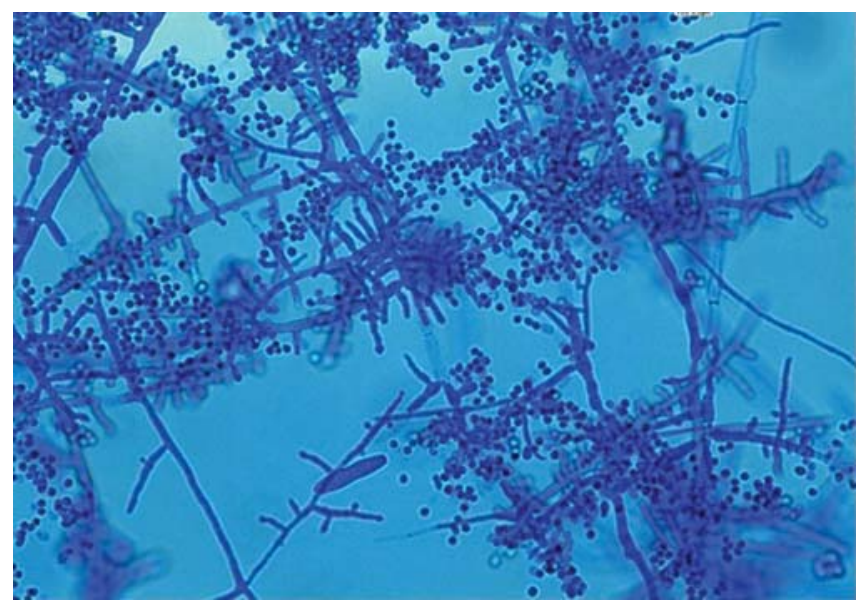

Figure 6: Lactophenol cotton blue mount of $T$. mentagrophytes viewed under the magnification $40 x$.

the most common age group affected by chronic dermatophytosis was 40-50 years old, and, in those with the first episode of the infection, the most commonly affected age group was in their second decade of life. Thus, chronic dermatophytosis is more common in the middle-age group and this may be due to the more vigorous physical activity associated with their occupation and the waning level of immunity and other comorbidities prevalent in this age group.

Chronic dermatophytosis was more common in males, with a male-to-female ratio of 1.26:1. Similar findings were obtained in other studies $[5,6]$. The generally increased prevalence in males may be correlated with their involvement in heavy physical work, more frequent outdoor activities, and thus an increased risk of exposure to infections.

In our study, more than half of the cases (54.5\%) had large body surface area involvement, and some presented themselves with an erythroderma-like condition. Ohashi et al. reported a case of erythroderma caused by dermatophytosis [7]. The larger body surface area involvement may be correlated with the chronicity of the disease.

Also, a majority of the cases (72.4\%) had experienced the involvement of more than one body site. This is in accordance with a similar study by Pathania et al. [6] The most common presentation in our study was tinea corporis with tinea cruris, followed by tinea corporis alone. Thus, multiple-site involvement may be a risk factor for the chronicity of dermatophytosis. Also, the involvement of groins may be related to the wearing of tight-fitting clothes and an increased sweating rate, both of which create a moist environment, which is favorable to the persistence and growth of dermatophytes. Also, tinea unguium was present in $13.1 \%$ of the patients, and most of them were diabetic. Thus, the persistence of the fungus in these areas may be a source of a spreading action to other body sites.

In our study, we found a relatively high number of cases with tinea faciei $(14.5 \%)$, in comparison with previous studies, and most of these patients were adults. Tinea faciei used to be rare in adults. Vineetha et al., in a similar study, found only $4.3 \%$ cases of tinea faciei in chronic dermatophytosis patients [5]. Sivaprakasam and Govindan found $6.7 \%$ of their cases affected by tinea faciei [4]. Thus, there is a changing trend as far as the pattern of involvement of dermatophytes is considered.

Most of the cases (30.34\%) were manual workers. Other commonly affected groups were students, followed by 
housewives and army/police personnel. All these groups are engaged in heavy outdoor work and/or household activities and/or wearing tight-fitting clothes for long periods of time, which predisposes to perspiration. In a study by Sharma R et al., students and army personnel were most commonly affected, but they included both new and chronic cases [8].

The mean of the hours of sun exposure was 3.5, and a majority of the cases $(68.35 \%)$ were exposed to more than 2.5 hours of sunlight. Prolonged sun exposure precipitates sweating, which may predispose to the chronicity of the disease.

Around half $(50.35 \%)$ of the patients in our study preferred wearing tight-fitting synthetic clothing, such as jeans, leggings, etc., and these were infrequently washed. Such clothing are, once again, a predisposing factor for excessive sweating, and their infrequent washing creates a damp environment favorable to the proliferation of fungus.

The most common socioeconomic class in our study was upper middle, with $27.59 \%$ of the patients in this group. Another commonly affected class was upper lower $(26.21 \%)$, followed by lower middle, lower, and upper. Hosthota et al. reported that $57.3 \%$ of their cases belonged to low socioeconomic strata, but their study included both new and chronic cases [9]. The occurrence of a chronic disease in the higher socioeconomic group in our study might have been associated with a different lifestyle, as in wearing synthetic clothing, such as jeans and leggings, the use of modern toilet seats, the use of washing machines for washing clothes, when the chances of mixing clothes belonging to different family members is increased and, thus, the spread of infection is faster.

Although most of the patients had average personal hygiene, poor personal hygiene, with infrequent washing of clothes and taking baths less often, was present in $20 \%$ of the cases. A history of fomite sharing with other family members was present in $74.48 \%$ of the cases, and a majority $(55.17 \%)$ lived in overcrowded conditions. All these factors were reported to be responsible for the chronicity and recurrence of dermatophytosis in previous studies [6].

A history of similar complaints in other family members was present in $43.45 \%$ of the cases, which may be due to fomite sharing, sharing of footwear, and mixing of clothes in washing machines. Untreated family members are, in turn, a source of reinfection, which may be a cause of the chronicity.

The most common comorbidity in our study was diabetes, present in $8.3 \%$ of the cases. This is in accordance with a study by Vineetha et al., in which diabetes was present in $11 \%$ of the chronic cases [5], and a much higher prevalence $(30 \%)$ was reported by Sivaprakasam and Govindan [4]. Other associated comorbidities included hypertension, hypothyroidism, atopic dermatitis, bronchial asthma, and anemia. Osteoarthritis, palmoplantar psoriasis, and pemphigus vulgaris were present each in $1(0.69 \%)$ patient. Patients with bronchial asthma were on oral and inhalational steroids, the patient with palmoplantar psoriasis was on methotrexate, and the patient with pemphigus vulgaris was on high-dose steroid pulse therapy and cyclophosphamide.

We know that atopics have a defective tilt of immunity toward TH2 cytokines and predispose to chronic dermatophytosis. In our study, atopy was present in $4.14 \%$ of the patients while $11.03 \%$ had atopic diathesis among family members. The absolute eosinophil count was high $(>450$ cells/ $\mu \mathrm{L})$ in $26.07 \%$ of the patients.

There is a general lack of awareness regarding the use and side effects of topical steroids among various non-dermatologists and medical practitioners, as demonstrated in a study by Abrol and Sharma [10]. A considerable number of patients $(78.62 \%)$ in our study had been applying topical antifungals that contained corticosteroids. Vineetha et al. also reported the use of topical steroid/antifungal creams by their patients, but the percentage was lower $(63 \%)$ when compared with our study [5]. Some of the patients had been applying plain topical potent corticosteroids as well. Corticosteroids suppress the signs and symptoms of inflammation, but dermatophytes continue to grow, leading to the subsequent flare of the disease [11]. Some of the patients $(6.21 \%)$ had also used oral steroids during the course of the disease. Thus, the use of corticosteroids may be the cause of the chronicity in these patients.

$73.10 \%$ of the patients in our study had been using oral antifungals in the form of fluconazole, itraconazole, terbinafine, or griseofulvin. However, in most cases $(61.38 \%)$, the dose and the duration of therapy were inadequate. The patients discontinued the medicine without medical advice as soon as they felt better. Non-compliance is a major cause of 
concern for a dermatologist as far as the treatment of dermatophytosis is concerned. Thus, proper education of the patients on the dose and duration of therapy may prove helpful.

In our study, $\mathrm{KOH}$ scraping was positive for fungal hyphae in $91.03 \%$ of the patients. A high positivity rate may be attributed to a high organism load in chronic cases, while the $\mathrm{KOH}$ negativity in some may be attributed to the minimal scaling present in the lesions.

The fungal culture was positive in $44.83 \%$ of the cases. However, Pathania et al. were able to grow dermatophytes in $60 \%$ of the cases [6]. A lower culture positivity rate in our study might have been due to more frequent antifungal use by chronic cases. Vineetha et al. were able to isolate dermatophytes in only $12 \%$ of cases of chronic dermatophytosis [5].

The most frequent isolate in our present study was Trichophyton mentagrophytes (53.85\%), followed by Trichophyton rubrum (38.46\%), Trichophyton tonsurans (4.61\%), and Microsporum canis and Trichophyton verrucosum ( $1.54 \%$ each). Most of the previous studies conducted in India have found Trichophyton rubrum the most common isolate $[4,12,13]$. Recently, however, several researchers have reported

Table 1: Distribution of the patients according to the pattern of involvement

\begin{tabular}{lcc}
\hline Pattern of involvement & No. of patients & Percentage \\
\hline Multiple-site involvement & 105 & 72.4 \\
Tinea corporis alone & 23 & 15.9 \\
Tinea cruris alone & 17 & 11.7 \\
\hline
\end{tabular}

Table 2: Distribution of the patients according to the fungal isolates

\begin{tabular}{lcc}
\hline Fungus isolate & No. of patients & Percentage \\
\hline Trichophyton mentagrophytes & 35 & 53.8 \\
Trichophyton rubrum & 25 & 38.4 \\
Trichophyton tonsurans & 3 & 4.6 \\
Microsporum canis & 1 & 1.5 \\
Trichophyton verrucosum & 1 & 1.5 \\
Total & 65 & 100 \\
\hline
\end{tabular}

Table 3: Risk factors

\begin{tabular}{lc}
\hline Risk factors assessed & Percentage of patients \\
\hline Manual workers & 30.3 \\
More than 2.5 hours of sun exposure & 68.3 \\
Synthetic clothing & 50.4 \\
Fomite sharing & 74.5 \\
Family history of dermatophytosis & 43.5 \\
Comorbidities & 23.4 \\
Extensive body area involvement (> 20\%) & 54.5 \\
Multiple-site involvement & 72.4 \\
Steroid application & 78.6 \\
Inadequate treatment & 61.4 \\
Absolute eosinophil count above 450 & 26.2 \\
\hline
\end{tabular}

Trichophyton mentagrophytes to be the predominant isolate $[8,14,15]$. Thus, there is a changing trend as far as the pathogenic organism of dermatophytosis is considered. Trichophyton mentagrophytes is associated with more widespread and inflammatory lesions. Also, some researchers have demonstrated its ability to survive longer on skin scales as compared to Trichophyton rubrum [16].

\section{CONCLUSION}

Our study recognized Trichophyton mentagrophytes as the predominant organism and tinea corporis with tinea cruris as the most common clinical presentation. Several risk factors that may contribute to the chronicity of dermatophytosis were identified in the majority of the patients. These included lack of proper hygiene, fomite sharing, wearing tight-fitting synthetic clothes, the presence of an infective focus in the family, prolonged sun exposure, the involvement of a larger body surface area, atopic diathesis, comorbidities, the use of topical steroids, and non-compliance to treatment. Thus, identifying and modifying the modifiable risk factors may be helpful in routine clinical practice.

\section{Statement of Human and Animal Rights}

All the procedures followed were in accordance with the ethical standards of the responsible committee on human experimentation (institutional and national) and with the 2008 revision of the Declaration of Helsinki of 1975.

\section{Statement of Informed Consent}

Informed consent for participation in this study was obtained from all patients.

\section{REFERENCES}

1. Weitzman I, Summerbell RC. The dermatophytes. Clin Microbiol Rev. 1995;8:240-59.

2. Dogra S, Uprety S. The menace of chronic and recurrent dermatophytosis in India: Is the problem deeper than we perceive? Indian Dermatol Online J. 2016;7:73-6.

3. Panda S, Verma S. The menace of dermatophytosis in India: The evidence that we need. Indian J Dermatol Venereol Leprol. 2017;83:281-4.

4. Sivaprakasam K, Govindan B. A clinicomycological study of chronic dermatophytosis of more than years duration. Int J Sci Res. 2016;5:551-4.

5. Vineetha M, Sheeja S, Celine MI, Sadeep MS, Palackal S, Shanimole PE, et al. Profile of dermatophytosis in a tertiary care center. Indian J Dermatol. 2018;63:490-5.

6. Pathania S, Rudramurthy SM, Narang T, Saikia UN, Dogra S. A prospective study of the epidemiological and clinical patterns of recurrent dermatophytosis at a tertiary care hospital in India. Indian 


\section{www.odermatol.com}

J Dermatol Venereol Leprol. 2018;84:678-84.

7. Ohashi T, Irie K, Yamamoto T. Erythroderma induced by dermatophytes. Our Dermatol Online. 2020;11:319-20.

8. Sharma R, Adhikari L, Sharma RL. Recurrent dermatophytosis: A rising problem in Sikkim, a Himalayan state of India. Indian J Pathol Microbiol. 2017;60:541-5.

9. Hosthota A, Gowda T, Manikonda R. Clinical profile and risk factors of dermatophytoses: a hospital based study. Int J Res Dermatol. 2018;4:508-13.

10. Abrol S, Sharma R. Knowledge, attitude, and behavior in the prescription of topical steroid for dermatological disorders among medical practitioners. Our Dermatol Online. 2020;11:357-9.

11. de Freitas RS, Neves PS, Charbel CE, Criado PR, Nunes RS, SantosFilho AM, et al. Investigation of superficial mycosis in cutaneous allergy patients using topical or systemic corticosteroids. Int J Dermatol. 2017;56:e194-8.

12. Surendran K, Bhat RM, Boloor R, Nandakishore B, Sukumar D. A clinical and mycological study of dermatophytic infections. Indian J Dermatol. 2014;59:262-7.
13. Puri N. Onchomycosis - a clinical and mycological study of 75 cases. Our Dermatol Online. 2012;3:172-7.

14. Noronha TM, Tophakhane RS, Nadiger S. Clinico-microbiological study of dermatophytosis in a tertiary-care hospital in North Karnataka. Indian Dermatol Online J. 2016;7:264-71.

15. Singh BSTP, Tripathy T, Kar BR, Ray A. Clinicomycological study of dermatophytosis in a tertiary care hospital in eastern India: A cross-sectional study. Indian Dermatol Online J. 2020;11:46-50.

16. Hosseinpour L, Zareei M, Borjian Boroujeni Z, Yaghoubi R, Hashemi SJ. Survival of dermatophytes in skin scales after 10 years storage. Infect Epidemiol Microbiol. 2017;3:96-9.

Copyright by Rabiya Bashir, et al. This is an open access article distributed under the terms of the Creative Commons Attribution License, which permits unrestricted use, distribution, and reproduction in any medium, provided the original author and source are credited.

Source of Support: Nil, Conflict of Interest: None declared. 